\title{
Aprendizaje-Servicio en escenarios digitales de aprendizaje: propuesta innovadora en la educación superior
}

\section{(Learning-Service in digital learning scenarios: innovative proposal for higher education)}

\author{
Marta Ruiz-Corbella \\ Juan García-Gutiérrez \\ Universidad Nacional de Educación a Distancia, UNED (España)
}

DOI: http://dx.doi.org/10.5944/ried.23.1.24391

\section{Cómo referenciar este artículo:}

Ruiz-Corbella, M., y García-Gutiérrez, J. (2020). Aprendizaje-Servicio en escenarios digitales de aprendizaje: propuesta innovadora en la educación superior. RIED. Revista Iberoamericana de Educación a Distancia, 23(1), pp. 183-198. doi: http://dx.doi.org/10.5944/ried.23.1.24391

\section{Resumen}

El reconocimiento y el rediseño de los aprendizajes en diferentes escenarios de aprendizaje son los principales desafíos a los que se enfrenta actualmente la educación superior. Una de las líneas de trabajo es el impulso de metodologías innovadoras, como el aprendizajeservicio (ApS), capaces de crear experiencias prácticas reales que cierren la brecha entre el aprendizaje que se genera en el aula y su aplicación en el mundo real. Metodologías que no pueden quedar al margen de las innovaciones tecnológicas, que no se entienden sin la información que proporcionan y sin la comunicación que es capaz de facilitar. Por primera vez, la transformación afecta a lo más genuino de todo ser humano: su estructura comunicativa y cognitiva desarrollado a través de un lenguaje multimodal, hipertextual y no lineal, lo que exige la transformación de la educación, a la vez que su apertura a los escenarios digitales. En este proceso, este artículo revisa los supuestos de esta apuesta por la innovación en la cual la tecnología posibilita esas nuevas formas de aprendizaje. En este marco, el ApS virtual integra las tecnologías en su diseño y desarrollo posterior, apoyándose en los recursos digitales que nos facilita este medio. Es decir, aprendizaje y servicio se desarrollan en y con la red, generando una modalidad inmersiva y real en el ciberespacio. Es necesario analizar lo que suponen estos nuevos escenarios educativos, en los que se incluyen estas metodologías que, al contacto con las tecnologías digitales, multiplican y amplifican sus posibilidades y efectos pedagógicos. 
Palabras clave: aprendizaje-servicio virtual; innovación educativa, escenarios digitales de aprendizaje; movilidad del estudiante; internacionalización; educación superior.

\begin{abstract}
The recognition and the redesign of learning in different scenarios are the main challenges for higher education. Therefore, methodologies are promoted, such as service-learning (SL), capable of creating real experiences, closing the gap between the learning generated in the classroom and the real world. Now, this transformation can not be left out of technological innovations, which are not understood without the information which it provides and without the communication that it is able to facilitate. For the first time, the transformation affects the most genuine of human being: its communicative and cognitive structure developed through a multimodal, hypertextual and non-linear language, which requires the transformation of education, as the same time as its opening up to digital scenarios. In this process, this paper reviews the assumptions of this commitment to innovation in which technology enables these new forms of learning. In this framework, virtual SL integrates the technologies in its own design, it relies on digital resources and the elements that this medium provides us. That is, learning and service are developed in and with the network, facilitating a proposal immersed in cyberspace. It is necessary to analyze these new educational scenarios in which these methodologies are included that, in contact with digital technologies, multiply and amplify their possibilities and pedagogical effects.
\end{abstract}

Keywords: E-service-learning; educational innovation; digital learning scenarios; student mobility; internationalization; higher education.

El último Informe Horizon 2019 (EDUCAUSE Learning Initiative, 2019) destaca, como propuestas educativas imprescindibles a corto plazo para la educación superior, el reconocimiento de los aprendizajes en diferentes escenarios y el rediseño de los espacios de aprendizaje avalados por un denominador común: el fomento del aprendizaje que denominan auténtico. Es decir, favorecer metodologías educativas, como el aprendizaje basado en proyectos, en competencias o el aprendizaje-servicio (ApS), capaces de crear experiencias prácticas reales donde los estudiantes aportan y construyen conocimiento, a la vez que desarrollan competencias específicas como futuros profesionales. En cada una de estas metodologías cada estudiante se convierte en promotor de su aprendizaje y, al mismo tiempo, productor de conocimiento, trasladándose el control de ese proceso del profesor al propio estudiante. De este modo, se incorpora el aprendizaje en entornos reales, a la vez que se favorece una cultura creadora y cívica en la educación superior, lo que colabora en que los estudiantes se impliquen activamente y contribuyan al desarrollo del ecosistema del conocimiento y a la mejora de situaciones reales del entorno en el que trabajan y/o viven (Adams Becker, Brown, Dahlstrom, Davis, DePaul, Diaz y Pomerantz, 2018).

De las metodologías mencionadas, este trabajo se centra en el ApS en una de sus aplicaciones o modalidades menos conocidas: el aprendizaje-servicio virtual 
(ApSv). El aporte clave de toda estrategia vinculada a esta metodología es su tensión por cerrar la brecha entre el aprendizaje que se genera en el aula y su aplicación al mundo real, orientada a atender eficazmente necesidades de una comunidad o grupo determinado, a la vez que integrada en la planificación de los contenidos curriculares, con el objetivo de optimizarlos (Strait y Sauer, 2004; Tapia, 2008). A partir de estas coordenadas se entiende que las estrategias y escenarios del ApS se desarrollan en contextos muy heterogéneos, implican a diversos grupos y/o individuos de una comunidad, a diferentes instituciones, organizaciones, etc. y ello con un diseño temporal también diferente y variable. Ahora bien, esta complejidad no puede llevarnos a admitir como ApS cualquier tipo de metodología basada en la experiencia, ya que únicamente se reconoce esta estrategia cuando surge

- una relación directa con los contenidos de una o varias asignaturas;

- un diseño pedagógico que responde a una necesidad, un problema, o un interés de una comunidad;

- una participación activa e implicada de los estudiantes y de la comunidad a la que se dirige;

- una práctica reflexiva (Bringle y Hatcher, 1999).

Por tanto, sintéticamente, se trata de una metodología que responde a las tres dimensiones que ya identificó Pestalozzi (Soëtard, 1994): cabeza (cognitiva), corazón (afectiva) y manos (acción), fórmula que promueve el ApS en todas sus propuestas.

\section{DEL APRENDIZAJE-SERVICIO PRESENCIAL AL VIRTUAL}

Partimos de la idea de que si nuestra sociedad nos va a exigir comunicar, producir y difundir con diferentes lenguajes, favoreciendo esa inteligencia colectiva que se desprende de la web 2.0 y 3.0 (Levy, 2004), las propuestas metodológicas de ApS cobran sentido ya que favorecen la creación de conocimiento, la interacción en la sociedad desde escenarios reales al servicio responsable a la sociedad. Estos proyectos, mayormente presenciales, no pueden desarrollarse ya ajenos a las tecnologías y ello desde dos perspectivas. Una es meramente instrumental, porque las tecnologías digitales ayudan y facilitan el desarrollo y su implementación. La segunda, más compleja, apunta a la propia experiencia de aprendizaje, ya que en una sociedad red aprender desde la experiencia implica experimentar las tecnologías. Por tanto, atender a los factores tecnológicos que posibilitan el desarrollo de diferentes modalidades del ApS implica preguntarse, de alguna manera, por la forma en que estas pueden intervenir o ser usadas en sus proyectos. $\mathrm{Y}$ al observar el rango de interacciones que se establecen, identificar los niveles o modos de interacción entre diseños tecnológicos y proyectos de ApS que favorecen, así, los diferentes procesos educativos. 
En esta línea, las tecnologías facilitan la propuesta de proyectos de ApS, a la vez que favorecen el desarrollo de proyectos innovadores que proveen nuevas modalidades de esta metodología. De acuerdo con lo expuesto en el apartado anterior, las tecnologías digitales se incluyen en los proyectos de manera instrumental al facilitar y optimizar su desarrollo, pero también pueden ser el objeto central del aprendizaje y/o del servicio, es decir, de la experiencia. Un ejemplo de ambas situaciones es la utilización de una página web o un blog para recoger los avances del proyecto de ApS que se está desarrollando, para difundirlo y/o para estar conectados e informados todos los participantes de dicha propuesta. En este caso, se trata de un proceso de evolución en el que se utiliza los recursos que ofrece la web para conectar, informar, difundir, etc. proyectos de diversa índole. Las tecnologías se integran en los diseños de ApS facilitando su gestión. La introducción no se establece con una intencionalidad educativa, sino puramente instrumental o facilitadora (Diaz-Corro, 2018; Gasper-Hulvat, 2018; Graves, 2018) (integración básica).

Sin embargo, también puede haber proyectos en los que las tecnologías pasan a ser el objeto de aprendizaje o bien de la prestación del servicio. Por ejemplo, proyectos como cibermanagers (Pantallas Amigas, 2019), donde la intención es formar(se) en el uso responsable de las redes sociales. O proyectos en los que estudiantes de electrónica o de informática reparan ordenadores y dispositivos para colectivos vulnerables; o ayudan a personas mayores a familiarizarse con las tecnologías y a utilizar aplicaciones como Skype, Whastapp o el correo electrónico. Todos estos casos recogen propuestas en las que lo instrumental es el objeto pedagógico (Lorenzo y Lorenzo, 2019; Salama, Awang Iskandara, Hanani Abang y Shoaib Farooqb, 2019) (inclusión intencional).

Ahora, se debe dar un paso más y pensar en proyectos de ApS en los que la tecnología favorezca procesos de inmersión. Estas no sólo deben estar integradas con una intencionalidad educativa, sino que el mismo proyecto está diseñado desde la perspectiva digital, apoyado en recursos digitales y con los elementos que facilita este medio. Esto es, tanto el aprendizaje como el servicio se desarrollan en y con la red, lo que evidencia una propuesta inmersa en el ciberespacio. Un ejemplo de esta modalidad es el proyecto “Español en vivo” (García-Gutiérrez, Ruiz Corbellay del Pozo Armentia, 2017), donde el aprendizaje y el servicio se desarrolla íntegramente en la red mediante la interacción virtual. Se trata, como lo denominan algunos autores, de un tipo de aprendizaje-servicio extremo (Waldner, McGorry y Widener, 2012; Yusof, Azean Atan, Harun y Doulatabadi, 2019), dado que tanto los aprendizajes como el servicio se desarrollan enteramente en el ciberespacio (inmersión tecnológica). El incremento de las interacciones y, en suma, de todas las facetas de la vida en la red hace de esta modalidad un recurso clave para el futuro de esta metodología, que acaba de empezar a desplegar todo su potencial pedagógico. Por tanto, es necesario analizar y ver de qué manera los nuevos escenarios educativos pueden (y deberían) incluir este tipo de metodologías que, si bien más extendidas en los formatos presenciales, al contacto con las tecnologías digitales multiplican y amplifican sus 
posibilidades y efectos pedagógicos. Sin duda, el surgimiento de un nuevo paradigma educativo en el que lo digital va a ser el elemento clave de esta interacción, por lo que las metodologías no pueden permanecer ajenas a ello (Waldner et al., 2012; Stirtz, 2019) (figura 1).

Figura 1. Evolución escenarios de intervención del ApS.

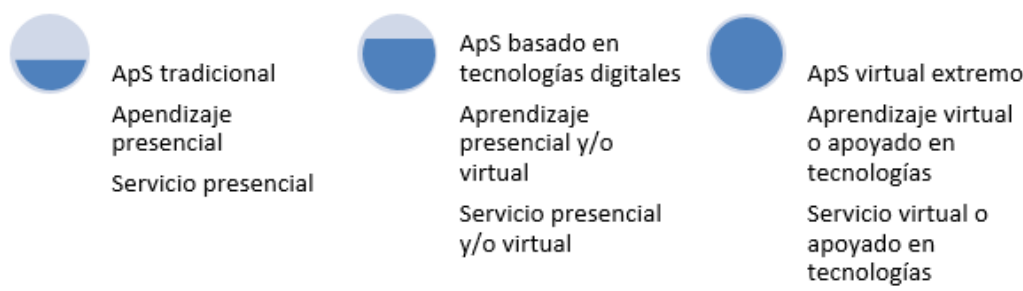

Fuente: adap. Yusof, Azean, Harun y Doulatabadi, 2018

Tras lo expuesto en los dos primeros apartados de este trabajo, la llegada al ApSv no es casual sino que refleja la progresiva virtualización de los procesos de enseñanza y aprendizaje. En efecto, el ApSv no supone una mera digitalización de procesos que también se podían realizar de manera presencial y que ahora, gracias a las tecnologías, se llevan a cabo virtualmente. Supone un paso más y requiere pensar el proceso de aprendizaje no como trasposición del presencial, sino reflexionar expresamente como sería su desarrollo en un ambiente virtual, en el que se plasma la singular y permanente continuidad online - offline.

Aunque las primeras experiencias con el ApSv no dejan de ser inductivas y basadas en la experiencia, es cierto que la amplia literatura que aborda esta modalidad del ApS construye también un marco de referencia ineludible. En efecto, destacan algunos trabajos que tratan de conceptualizar y clarificar qué sucede cuando el ApS llega a los estudiantes online (Strait y Sauer, 2004; Waldner et al., 2012), especialmente en la educación a distancia (Guthrie y McCracken, 2010, 2014), estudiando la particular forma de mediación comunicativa que se produce en este tipo de procesos (Bourelle, 2014; Harris, 2017). Algunos autores avanzan también en el análisis específico que ofrece esta nueva modalidad y las diversas maneras en que se manifiesta la combinación entre los procesos de aprendizaje y servicio online y presenciales (Waldner, McGorry y Widener, 2010).

Desde esta misma perspectiva, también aparecen trabajos que abordan aspectos y temáticas específicas como, por ejemplo, el papel de las redes sociales en los proyectos de ApS (Moeller y Nagy, 2013), análisis de proyectos que desarrollan tutorías en línea (ChanLin, 2016), los estudios sobre liderazgo (Goertzen y Greenleaf, 2016; Purcell, 2017), o el desarrollo ético (Ruso, 2012). Además, como no podía ser de otra forma, en toda propuesta de ApSv es preciso insistir en cómo favorecer y 
potenciar el pensamiento reflexivo y crítico de los estudiantes (Guthrie y McCracken, 2014).

Por otra parte, no debe obviarse otras experiencias en el medio virtual que sirven para apuntalar la modalidad virtual del ApS. En concreto, nos referimos a las prácticas virtuales y las propuestas de movilidad virtual (Ruiz-Corbella y Álvarez González, 2014). En ambas situaciones estos proyectos acercan la posibilidad de realizar, como pretende el ApS, un tipo de aprendizaje práctico real, no simulado, en un espacio virtual de aprendizaje. Por su parte, la movilidad virtual aproxima a todos los estudiantes las ventajas y beneficios que tiene la movilidad académica internacional (Crabtree y Sapp, 2018). En este sentido, adoptar este enfoque permite el acceso y el intercambio entre estudiantes de diferentes nacionalidades sin necesidad de una presencialidad en un entorno geográfico determinado. En el caso de la movilidad virtual, este tipo de estancias permite a los estudiantes no abandonar sus responsabilidades cotidianas, y evita las restricciones de cualquier tipo, especialmente de tiempo, para llevarla a cabo, adecuándola a cada situación personal (Ruiz Corbella y García Aretio, 2010).

\section{APRENDIZAJE-SERVICIO EN ESCENARIOS DIGITALES: El caso del proyecto "Español en vivo"}

Sin duda, estamos ante una amplia generalización e implantación de la metodología de ApS en las instituciones universitarias, aunque aún no ha alcanzado de forma significativa a las universidades a distancia y/o virtuales. Algunos advierten que estas instituciones quedan fuera de su ámbito de aplicación, al estar sus estudiantes ubicados en diferentes localizaciones geográficas y la interacción en los procesos de enseñanza-aprendizaje sea únicamente a través de la red, por lo que plantear acciones de intervención solidaria se reconocen como poco factibles. A la vez señalan que el perfil de los estudiantes de instituciones educativas virtuales es mayoritariamente adulto, con responsabilidades laborales y familiares, por lo que el desarrollo de cualquier actividad, fuera de lo estrictamente diseñado por cada asignatura y/o titulación, resulta sumamente complejo.

Sin embargo, consideramos que los estudiantes de universidades no presenciales, al igual que sucede con los de cualquier otra institución, no deberían permanecer al margen de las innovaciones pedagógicas ya que, en primer lugar, todo estudiante, ya sean en la enseñanza presencial o a distancia, debería estar familiarizado con las innovaciones pedagógicas de su tiempo. En segundo lugar, si en todas las áreas vitales avanzamos hacia escenarios híbridos online - offline, también su formación debería darse en estos mismos contextos. Y, por último, las instituciones educativas que interaccionan en la red deben comprometerse con el desarrollo de competencias éticas y cívicas y, por tanto, buscar vías para su desarrollo en el espacio que precisamente utilizan para la formación. 
Como respuesta a esta demanda formativa en un escenario digital, se diseñó el proyecto de ApSv "Español en vivo", centrado en la competencia intercultural y de ciudadanía global, a partir de la mejora de la competencia de expresión oral entre estudiantes de las Facultades de Educación de la Universidad Nacional de Educación a Distancia - UNED (España), los de la Ecole Normale Supérieure (ENS) de Porto Novo (Benin), los de Strathmore University (SU) de Nairobi (Kenia) y, los últimos en unirse al proyecto fueron los de la Dschang University (Camerún). Los estudiantes de la UNED se forman como futuros educadores sociales y los africanos cursan el español como segunda lengua y, en algunos casos, como profesores de nuestro idioma. Este proyecto se desarrolla a través de conversaciones y entrevistas en línea, donde los alumnos africanos de estas instituciones tienen la oportunidad de practicar el castellano hablado con nativos, y los estudiantes españoles profundizan en los contenidos de sus asignaturas desde una perspectiva intercultural y de ciudadanía global. Esta experiencia se desarrolla íntegramente en el ciberespacio, apoyándose en recursos digitales que facilitan esta interacción -Skype, Hangout (Google) o Whastapp-, que cada grupo escoge de acuerdo a sus posibilidades de acceso a la red y de dispositivos. Por otro lado, toda la información sobre el proyecto se encuentra disponible en una web específica, en la que se incorporan las buenas prácticas para la realización del proyecto, los videos con los que los estudiantes africanos se presentan, los cuadernos de campo intercultural (CCI) que cada alumno realiza, enlaces, etc.

En este proceso de interacción entre estudiantes de diferentes regiones, resulta fundamental la reflexión (Páez y Puig Rovira, 2013; Pacho, 2018). La pregunta de partida es simple: ¿qué pretendemos lograr con este proyecto de ApSv? ¿Cómo se facilita ese proceso, qué recursos se deben utilizar? ¿Qué incidencia formativa tienen? En primer lugar, que los estudiantes reflexionen sobre su propia experiencia de aprendizaje es una condición indispensable de cualquier proceso educativo, pero especialmente refuerza la calidad de todo proyecto de ApS (Bringle y Hatcher, 1999; Bowen, 2007; Faszer-McMahon, 2013; Bursaw; Kimber y Carrington, 2015). Además de contribuir al desarrollo del pensamiento crítico (Mendoza Guerrero, 2016).

Desde la experiencia del proyecto, se considera el que los estudiantes reflexionen sobre su actuación en este proyecto, les ayuda a profundizar y (re)conocer esta metodología y lo que puede aportar a su futuro como profesionales de la educación. Constituye la manera de hacer realmente suyos los aprendizajes alcanzados. Volviendo a recorrer el camino, descubren implicaciones o elementos que pasaron desapercibidos durante su desarrollo, son capaces de comprender mejor en qué consiste y qué alcance puede tener esta metodología para, posteriormente, replicarla en sus lugares de trabajo. Además de valorar sobre lo que ha supuesto para ellos esa experiencia en la red con pares de contextos tan distantes y diferentes.

La segunda razón, por la cual es importante la reflexión, se centra en evitar sesgos o manipulaciones en los estudiantes. El ApS es una metodología basada y que promueve valores, compromiso cívico y responsabilidad. Ahora bien, interesa que 
sean los propios estudiantes quienes sean capaces de dar sentido a la experiencia realizada y desarrollar un pensamiento crítico. Además, la centralidad del estudiante es uno de los rasgos característicos de estos proyectos. En este punto, resaltamos que la reflexión juega un doble papel. En primer lugar, como prevención del adoctrinamiento y, en segundo lugar, como un momento donde el estudiante (re) configura su aprendizaje y el sentido ético-cívico de la propia experiencia realizada. En efecto, la falta de reflexión, o los impedimentos para que se produzca, constituye una de las características más destacadas de los procesos de adoctrinamiento en los que se obstaculiza la capacidad de autorreflexión, en aras de un aprendizaje basado más en la emoción. Siendo los proyectos de ApS una experiencia pedagógica global e inmersiva, donde entra en juego también lo afectivo, es preciso extremar el cuidado de que los estudiantes sean capaces de encontrar espacios y momentos para desapegarse de la experiencia realizada y valorar la experiencia desde argumentos reflexivos.

En esta fase del proceso, los estudiantes deberían ser capaces de (re)organizar la experiencia realizada en tres direcciones: 1 - saber exponer sus propias ideas y razones sobre la experiencia realizada; 2- poder identificar aquellos valores significativos y movilizar su acción de manera coherente con ellos; 3- interiorizar los aprendizajes no tanto como contenidos sino como formas de aproximarse a la realidad, en otras palabras, ver el conocimiento no sólo como maletas llenas, sino como "algo que nos permite viajar con una visión diferente" (Esteve Zarazaga, 2012, p. 48). De lo dicho hasta ahora, pudiera parecer que la reflexión constituye el momento final del proyecto, como si los estudiantes hicieran una valoración final de la experiencia realizada. Sin embargo, esta es necesaria como lo que algunos denominan reflexiónen-la-acción. Esto es, facilitar elementos para que la reflexión sea una constante a lo largo del proyecto, de inicio a fin, de manera que los estudiantes sean capaces de apropiarse de la experiencia según la realizan y no lo vean sólo como un mero ejercicio para la evaluación (Bukas, Azean, Farhana, Junaidi y Haruzuan, 2018).

Por otra parte, es preciso también que los estudiantes interioricen el sentido y el alcance que tiene, en este caso, la ciudadanía global en el contexto de diálogo académico propuesto por el proyecto. No se trata de ofrecer una definición previa, sino que sean los propios estudiantes quienes, tras la experiencia realizada, puedan argumentar qué significa para cada uno de ellos los aprendizajes adquiridos.

La metodología, que se ha considerado más apropiada para activar la reflexión en este proyecto, ha sido la biográfico-narrativa (Moriña, 2017), a modo de diario personal o diario de campo. Concretamente, se ha elaborado un instrumento que permite llevar a cabo tanto la recogida de información necesaria para la evaluación como para facilitar la reflexión de los estudiantes durante todas las fases del proyecto. Se trata del denominado Cuaderno de Campo Intercultural (CCI), que permite activar la reflexión y, al mismo tiempo, acceder al tipo de experiencia y a la adquisición de la competencia ética y el compromiso cívico en los términos establecidos en el proyecto (García-Gutiérrez, Fuentes y del Pozo, 2018). 
Los estudiantes también han dispuesto a lo largo del proceso de un canal de comunicación asíncrono específico donde compartir con el resto de compañeros sus impresiones, dudas, propuestas, etc., junto con la utilización de los grupos de Whatsapp donde comparten preguntas, se inician o continúan diálogos o, simplemente, se consensua la mejor fecha para la entrevista.

Si bien la narración de la experiencia en un contexto virtual podría adoptar diferentes formas de Digital Storytelling (Wexler, Flanders y Brexa, 2011), se ha optado por una forma más personal de contar la experiencia con este CCI, en el que se anima a los estudiantes a que incluyan imágenes, capturas de pantalla de su ordenador o teléfono móvil, enlaces a audios, que reflejan el itinerario recorrido -siempre tras el consentimiento de los participantes-. La razón principal tiene que ver con favorecer la sinceridad que se asocia al desarrollo de este diario. Ahora bien, no se considera que sean opciones excluyentes. El Digital Storytelling ofrece posibilidades interesantes para el ApSv que sería necesario explorar, al ser capaz de propiciar los procesos de reflexión online y generar productos que reflejen y den a conocer la experiencia realizada (Beirne, 2018).

\section{ALGUNOS RESULTADOS PRELIMINARES DEL PROYECTO}

Si bien en posteriores trabajos se avanzará en la lectura pedagógica de los CCI, en este trabajo adelantamos algunos resultados preliminares. Dado que el proyecto es aún muy joven, los CCI nos permitirán reflexionar e investigar sobre diferentes aspectos importantes del proyecto de innovación desde un enfoque cualitativo. Los temas, que abordamos, a continuación, tienen interés desde esta perspectiva, ya que la investigación en innovación resulta clave no sólo para mejorarlo, sino para que revierta realmente en el aprendizaje pretendido y en el desarrollo de competencias. En este sentido, nos interesa destacar dos aspectos. Por un lado, cómo perciben los estudiantes los objetivos del proyecto, lo que redundará no sólo en la mejora del mismo, sino también permitirá interrogarnos acerca de cómo perciben la formación académica y qué les aporta para su quehacer profesional. Por otro, los CCI informan de los aprendizajes en relación a cómo experimentan el compromiso cívico y la competencia ética. Concretamente, nos centraremos en cómo han elaborado la idea de ciudadanía global los 21 estudiantes que participaron en el proyecto durante este último curso 2018/19.

Uno de los resultados más relevantes, en el contexto de una universidad a distancia, ha sido ofrecer a los estudiantes la oportunidad de participar en un proyecto de ApS internacional, dado que, hasta el momento, quedaban reservados únicamente a las universidades presenciales. Facilitar esta opción en las universidades españolas ha sido una de las aportaciones más significativas de esta experiencia. Por tanto, y a pesar de que no todos los estudiantes matriculados en las asignaturas han participado en esta propuesta, sí hemos podido dar a conocer la metodología del ApSv entre todos ellos, a través de los cursos virtuales de las asignaturas, videos formativos, guías, etc.; contribuyendo así a su difusión. 
Por otro lado, consideramos relevante preguntarles, tal como acabamos de mencionar, cómo experimentan cada objetivo del proyecto en relación a dos categorías, la formación académica y el futuro desempeño profesional: (1) comunicar y exponer contenidos específicos de las asignaturas por vías y recursos virtuales; (2) mejorar el aprendizaje del español mediante una comunicación directa con hablantes nativos; (3) conocer diversas culturas pedagógicas instaladas en diferentes contextos internacionales; (4) profundizar en la noción de ciudadanía global y su relación con los derechos humanos y los Objetivos de Desarrollo Sostenible (ODS); (5) desarrollar el compromiso cívico de los estudiantes a través de la ciudadanía global, el diálogo intercultural como vías para superar los prejuicios y fomentar una cultura de paz; (6) ser capaz de asumir un compromiso ético a través de las tareas propias de la profesión; (7) familiarizar a los estudiantes con el diseño y la puesta en marcha de acciones pedagógicas innovadoras.

La pregunta presente en el CCI era sencilla: qué objetivos del proyecto son más relevantes para la formación académica y cuáles para el desempeño profesional (figura 3). Entre los resultados, cabe destacar que mejorar la comunicación oral en español no se ha valorado ni en lo académico ni en lo profesional, otorgando más relevancia para su formación a los otros objetivos, especialmente al compromiso cívico y al diálogo intercultural. Es importante, además, que los estudiantes no contemplen los proyectos de aprendizaje-servicio únicamente desde una perspectiva de la formación académica sino, sobre todo, desde un enfoque práctico, de acuerdo con la propia filosofía de estos proyectos, porque es lo que favorece al desarrollo de sus competencias profesionales.

Figura 2. Valoración de los objetivos del proyecto 'Español en vivo' por parte de los estudiantes. Curso 2018-2019

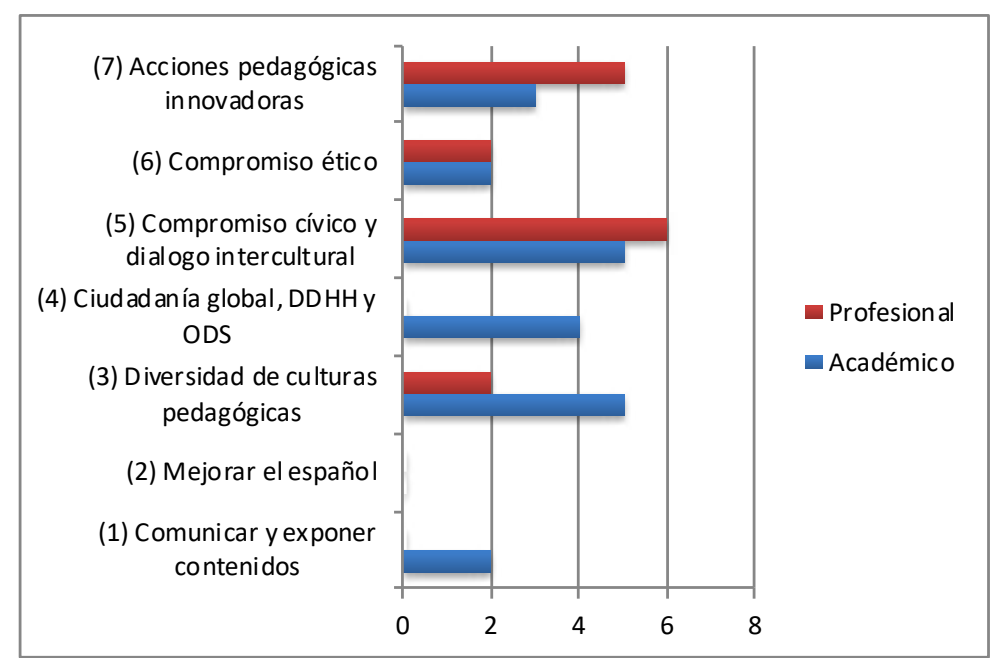


Por otro lado, si atendemos a la bibliografía reciente sobre la ciudadanía global, comprobamos un interés creciente (Martínez Lirola, 2019). Ahora bien, para nosotros no era tan importante la transmisión de una determinada imagen de ciudadanía global, si no la que ellos mismos pudieran experimentar y elaborar (figura 3). En los CCI se encuentran elementos interesantes que nos permiten acceder a cómo interiorizan este tipo de nociones, en principio, más teóricas, interpretaciones que se recogen en la siguiente figura.

Figura 3. Definiciones de ciudadanía global realizadas por los estudiantes. Curso 2018-2019

\section{Cómo entiendo la ciudadanía global}

Una ciudadanía donde todas las personas se respetan, conviven y se integran de manera pacífica. Un lugar donde se reconocen y respetan los derechos inherentes a todo ser humano". CCI 1

Forma de entender y actuar en la sociedad, en la que se busque una actitud proactiva hacia la consecución de igualdad de oportunidades, de derechos y obligaciones de todas las personas”. CCI 2

Entiendo CG como la ciudadanía del mundo, más allá de los estados o naciones. El proceso de globalización afecta a todas las esferas de la vida social, cultural, política y económica. Este proceso está dando lugar a un nuevo mundo globalizado con una nueva ciudadanía, que no necesariamente debe compartir un mismo espacio físico o país para actuar como ciudadano y disfrutar de sus derechos y ejercer sus obligaciones como ciudadano. Debe ser una ciudadanía participativa y respetuosa con la diversidad de todas las personas (origen, religión cultura) donde la diversidad sea considerada en positivo y no tenga connotaciones negativas. Una ciudadanía que tenga como actitudes y valores la solidaridad, la paz la tolerancia la justicia el consumo responsable, la participación y el firme compromiso en su vida de contribuir a construir un mundo más libre, diverso y equitativo. CCI3

Valor imperante en la sociedad en el que se trabaja por un mundo más equitativo. CCI 4 Entendimiento personal de que todas las personas somos iguales, somos ciudadanos de un mismo planeta, es decir que las personas estamos por encima de las fronteras y las nacionalidades. CCI 5

Corriente, como aquellas personas de la sociedad que se comprometen de forma activa a favorecer, a conseguir un mundo, una sociedad más justa, equitativa, sostenible posible, de acuerdo a unas normas, códigos ético morales y sin discriminar por razón de raza, género, sexo, etc. CCI 6

Desde mi punto de vista la ciudadanía global es una utopía realizable por la que todos los ciudadanos deberíamos de contribuir, esta utopía se basa en el compromiso activo para el logro de una sociedad más justa e igualitaria donde estén presentes de manera notable la equidad y la sostenibilidad. CCI 7

Un modelo de ciudadanía nuevo que busca más todo lo relacionado con la búsqueda de la equidad y la igualdad. CCI 8

Esa realidad que nos hace ser conscientes de que somos ciudadanos del mundo, habitantes de un mismo planeta. Realidad que nos recuerda que "la tierra es un solo país y la humanidad sus ciudadanos". CCI 9 


\section{Cómo entiendo la ciudadanía global}

Ciudadano del mundo, en donde uno se da cuenta de los diversos desafíos del mundo actual, reconoce sus derechos y los de los demás, que se indigna con las injusticias que acaecen en el mundo y que por ende intenta combatir la desigualdad existente entre los ciudadanos del mundo, y además es capaz de criticar las políticas económicas, sociales, culturales, tecnológicas y ambientales. CCI 10

A partir de estas explicaciones sobre cómo perciben o qué significa para los estudiantes la idea de ciudadanía global, comprobamos que, en primer lugar, este aspecto no ha quedado sólo en un objetivo teórico del proyecto, sino que algunos la han experimentado de forma concreta. Sobre todo, subrayando en muchos casos el elemento de "responsabilidad" y "compromiso" que implica (p.e., CCI 2, 7, 9 y 10). A la vez que se destacan diversas dimensiones de esta nueva ciudadanía, especialmente en relación a la sostenibilidad y a las condiciones de la vida en el planeta (p.e., CCI $1,5,7$ y 9 ).

La ciudadanía global constituye una temática altamente relevante para la educación superior. No sólo porque los traídos y llevados ODS hayan permeado -o, al menos, traten de impactar- las políticas universitarias, sino porque el mundo y la sociedad son globales e interdependientes y es preciso que la comprensión pedagógica sobre cómo formar a las nuevas generaciones de ciudadanos esté más cerca de los desafíos actuales y futuros que de los problemas decimonónicos que planteaba la constitución de los estados nación.

Sin duda, consideramos que el proyecto abre una nueva perspectiva en relación a cómo podemos entender la educación como futura actividad profesional, experimentando la red como un espacio de solidaridad y una vía para el desarrollo del compromiso cívico que, quizá, hasta el momento no se había planteado. Nos gustaría concluir con unas palabras del CCI de una estudiante de la UNED (España) que encierra muchos de los aprendizajes intangibles que pueden promover este tipo de proyectos:

"Me ha fascinado el símil de los chicos de Benín al comparar la educación con el agua. Una de las chicas comentaba 'Un país sin educación es un país sin agua'. Y, de sobra, es sabido que sin agua no hay vida”.

\section{REFERENCIAS}

Adams Becker, S., Brown, M., Dahlstrom, E., Davis, A., DePaul, K., Diaz, V., y Pomerantz, J. (2018). NMC Horizon Report: 2018 Higher Education Edition. Louisville, CO, EEUU: EDUCAUSE. Recuperado de https://bit.ly/2wrocSO
Beirne, H. K. (2018). A Storied Tale: Melding Digital Storytelling, Service-Learning, and Digital and Information Literacy Skills for Pre-Service Teachers. In Library Service and Learning: Empowering Students, Inspiring Social Responsibility. Kentucky, USA: ACRL Publications. 
Bourelle, T. (2014). Adapting servicelearning into the online technical communication classroom: a framework and model. Technical Communication Quarterly, 23(4), 247-264.

Bowen, G. (2007). Reflection in servicelearning. Evaluation/Reflection, 24. Recuperado de https://bit.ly/2xQr8sw

Bringle, R., y Hatcher, J. (1999). Reflection in Service Learning: Making Meaning or Experience. Evaluation/Reflection, 23 [Informe]. Recuperado de https://bit. ly/2JzpoL1

Bukas, V., Azean, N., Farhana, N., Junaidi, J., y Haruzuan, M. N. (2018). Improving Student's Learning Outcomes through E-Service Learning Based on Authentic Learning Strategy. Innovative Teaching and Learning Journal, 2(1), 8-16.

Bursaw, J., Kimber, M., y Carrington, S. (2015). Teaching Reflection for ServiceLearning. In M. E. Ryan (Ed.), Teaching Reflective Learning in Higher Education (153-169). Berlin, Germany: Springer.

ChanLin, L. (2016). Students' involvement and community support for service engagement in online tutoring. Journal of Educational Media \& Library Sciences, 53(2), 245-268.

Crabtree, R. D., y Sapp, D. A. (2018). International Service-Learning guiding theories and practices for Social Justice. In D. E. Lund, The Wiley International Handbook of Service-Learning for Social Justice. (319-352), Hoboken, N. J., USA: Wiley-Blackwell. doi: doi. org/10.1002/9781119144397.ch15.

Diaz-Corro, K. (2018). Service Learning Through Extracurricular Activities: Development and Implementation of a Transportation Engineering Learning Module. Civil Engineering Undergraduate Honors Theses. 49. Recuperado de https://bit.ly/2JEoLf2 EDUCAUSE Learning Initiative (2019). Informe Horizon 2019. Recuperado de https://bit.ly/2GQZv86
Esteve Zarazaga, J. M. (2012). Educar: un compromiso con la memoria. Barcelona: Octaedro.

Faszer-McMahon, D. (2013). Social Networking, Microlending, and Translation in the Spanish ServiceLearning Classroom. Hispania, 96(2), 252-263.

García-Gutiérrez, J., Ruiz-Corbella, M., y del Pozo, A. (2017). Developing Civic Engagement in Distance Higher Education: A Case Study of Virtual Service-Learning (vSL) programme in Spain. Open Praxis, 9(2), 235-244.

García-Gutiérrez, J., Fuentes, J. L., y del Pozo, A. (2018). Promoción de la competencia ética y el compromiso cívico y su evaluación en los proyectos de aprendizaje-servicio. En M. Ruiz-Corbella y J. García-Gutiérrez (Eds.), Aprendizajeservicio: los retos de la evaluación (117134). Madrid: Narcea.

Gasper-Hulvat, M. (2018). "More like a real human being": humanizing historical artists through remote ServiceLearning. Journal of Experiential Education, 41(4), 397-410. doi: doi. org/10.1177/1053825918808321.

Germain, M. L. (2019). Integrating ServiceLearning and Consulting in Distance Education. Bingley, UK, Emerald Publishing Limited.

Goertzen, B., y Greenleaf, J. (2016). A student-led approach to eServiceLearning: a case study on service project effectiveness within a fieldwork in leadership studies course. International Journal of Research on Service-Learning and Community Engagement, 4 ( 1 ), 119-135.

Guthrie, K., y McCracken, H. (2010). Making a difference online: facilitating servicelearning through distance education. Internet and Higher Education, 13, 153157.

Guthrie, K., y McCracken, H. (2014). Reflection: the importance of making 
meaning in e-service-leaning courses. Journal of Computing Higher Education, $26,238-252$.

Graves, N. A. (2018). Service-Learning in the hybrid classroom. Family Science Review, 22(3), 87-102.

Harris, U. (2017). Virtual partnerships: engaging student in e-serviceleaning using computer-mediated communication. Asia Pacific Media Educator, 27(1), 103-117. doi: dx.doi.org/10.18381/Ap.v10n1.1140.

Lévy, P. (2004). Inteligencia colectiva: por una antropología del ciberespacio. Washington, DC, USA: Organización Panamericana de la Salud. Recuperado de https://bit.ly/1fig1bH

Lorenzo, C., y Lorenzo, E. (2019). Opening Up Higher Education: An E-learning Program on Service-Learning for University Students. In W. Karwowski, T. Ahram, y S. Nazir, Advances in Human Factors in Training, Education, and Learning Sciences, (27-38). New York, USA: Springer.

Martínez-Lirola, M. (2019). Una propuesta didáctica para introducir la educación para la ciudadanía global en la enseñanza universitaria. Revista Electrónica Educare, 23(2), 1-20.

Mendoza Guerrero, P. L. (2016). La investigación y el desarrollo de pensamiento crítico en estudiantes universitarios. [Tesis Doctoral]. Málaga: Universidad de Málaga.

Moeller, M., y Nagy, D. (2013). More questions than answers: Assessing the impact of online social networking on service learning Project. International Journalfor the scholarship of teaching and learning, 7(1), 1-23.

Moriña, A. (2017). Investigar con historias de vida. Metodología biográficonarrativa. Madrid: Narcea.

Pacho, T. O. (2018). Service-Learning in Higher Education in Afrika. Newcastle, UK: Cambridge Scholars Publishing.
Páez, M., y Puig Rovira, J. M. (2013). La reflexión en el aprendizaje-servicio. Revista Internacional de Educación para la Justicia Social, 2(2), 13-32.

Pantallas Amigas (2019). Cibermanagers se consolida como proyecto de ciberconvivencia y ciudadanía digital necesario. [blog], 21 enero.

Purcell, J. (2017). Community-engagement pedagogy in the virtual classroom: integrating eService-Learning into online leadership education. Journal of Leardership Studies, 11(1), 65-70.

Ruiz Corbella, M., y García Aretio, L. (2010). Movilidad virtual en la educación superior: coportunidad o utopía? Revista española de pedagogía, 246, 243-259.

Ruiz-Corbella, M., y Álvarez González, B. (2014). Virtual Mobility as an Inclusion Strategy in Higher Education: Research on Distance Education Master Degrees in Europe, Latin America and Asia. Research in Comparative and International Education, 9(2), 165-180. doi: doi. org/10.2304/rcie.2014.9.2.165.

Ruso, N. (2012). The role of technology: community based service-learning projects on ethical development. The Turkish Online Journal of Educational Technology, 11(3), 375-385.

Salama, M., Awang Iskandara, D. N., Abang, D. H., y Shoaib Farooqb, I. M. (2019). Technology integration in servicelearning pedagogy: A holistic framework. Telematics and Informatics, 38, 257-273. doi: doi.org/10.1016/j.tele.2019.02.002.

Soëtard, M. (1994). Johan Heinrich Pestalozzi (1746-1827). Perspectivas: revista trimestral de educación comparada, XXIV(1-2), 299-313.

Stirtz, G. E. (2019). Online Professional Development in Academic ServiceLearning: Promoting Community Engagement in Public Education. Hershey, Pen, USA: IGI Global.

Strait, J., y Sauer, T. (2004). Constructing experiential learning for online courses: 
the birthof e-service, Educause Quarterly, $1,62-65$.

Tapia, N. (2008). Calidad académica y responsabilidad social: el aprendizaje servicio como puente entre dos culturas universitarias. En M. Martínez (Ed.), Aprendizaje servicio y responsabilidad social de las universidades (27-56). Barcelona: Octaedro.

Waldner, L., McGorry, S., y Widener, M. (2010). Extreme E-Service-Learning (XE$\mathrm{SL})$ : e-service learning in the $100 \%$ online course. MERLOT Journal of online learning and teaching, 6(4), 839-851.

Waldner, L., McGorry, S., y Widener, M. (2012). E-Service-Learning: the evolution of service learning to engage a growing online student population. Journal of Higher Education Outreach and Engagement, 16(2), 123-150.

Wexler, E., Flanders, D., Sullivan, M., y Brexa, J. (2011). Digital Storytelling within a service-learning partnership: technology as product and process for university students and culturally and linguistically diverse high school youth. In M. Bowdon y R. Carpenter (Eds.), Higher Education, Emerging Technologies, and Community Partnerships: Concepts, Models and Practices (pp. 88-105). Pennsylvania, USA: IGI Global, Hershey. Yusof, A., Azean, N., Harun, J., y Doulatabadi, M. (2018). Towards A Conceptual Framework for Service-Learning in Online Learning Environment. [paper] International Conference on Industrial Engineering and Operations Management. Bandung, Indonesia. Recuperado de https://bit.ly/2xMQeIT

Yusof, A., Azean, N., Harun, J., y Doulatabadi, M. (2019). Developing Students Graduate Attributes in Service Learning Project through Online Platform. [paper]. Proceedings of the International Conference on Industrial Engineering and Operations Management. Bangkok, Thailand. Recuperado de https://bit. $\underline{\mathrm{ly} / 2 \mathrm{GedMuN}}$

\section{PERFIL ACADÉMICO Y PROFESIONAL DE LOS AUTORES}

Marta Ruiz-Corbella. Profesora Titular de la Universidad Nacional de Educación a Distancia (España). Miembro del Grupo de Investigación Educación Superior Presencial y a Distancia (ESPYD) y del Grupo de Innovación Docente para el Desarrollo de la Competencia Ética y Cívica en la Educación Superior. Entre sus temas de interés destacan la educación moral y cívica, la educación superior, la formación del profesorado y la educación a distancia. ID: https://orcid.org/oooo0001-5498-4920

E-mail: mruiz@edu.uned.es

Juan García-Gutiérrez. Profesor Contratado. Doctor de Filosofía de la Educación de la Universidad Nacional de Educación a Distancia (España). Coordinador del Grupo de Innovación Docente para el Desarrollo de la Competencia Ética y Cívica en la Educación Superior. Delegado del Rector de la Oficina de Aprendizaje-Servicio de la UNED. Entre sus temas de interés destacan la filosofía de la educación, educación moral, tecnologías y educación ética.

E-mail: juangarcia@edu.uned.es 
Dirección:

Dpto Teoría de la Educación y Pedagogía Social

Facultad de Educación - UNED

c/ Juan del Rosal, 14

28040 Madrid (España)

Fecha de recepción del artículo: 17/05/2019

Fecha de aceptación del artículo: 14/06/2019

Fecha de aprobación para maquetación: 10/08/2019 